\title{
Higher order dilaton gravity: effective equations at the brane
}

\section{Dominika Konikowska*}

Institute of Theoretical Physics, University of Warsaw

Hoża 69, PL-00-681 Warsaw, Poland

E-mail: dominika.konikowska@fuw.edu.pl

\begin{abstract}
A higher order theory of dilaton gravity is motivated and presented. Although its Lagrangian density involves higher powers of the Riemann tensor and of the first two derivatives of the dilaton, the resulting equations of motion are quasi-linear in the second derivatives of both the metric tensor and of the dilaton. The higher order dilaton gravity with corrections up to arbitrary order in field derivatives is investigated in a co-dimension 1 brane scenario with general brane localized interactions. The derivation of the effective gravitational equations of motion at the brane is addressed in the covariant approach, with the results presented in detail for the first order dilaton gravity. The crucial influence of the bulk $\mathbb{Z}_{2}$ symmetry (with its fixed point at the brane position) on the form and the number of effective brane equations is shown and discussed. The generic influence of the bulk gravity solution on the effective Einstein-like equation at the brane is demonstrated. The resulting modified Friedmann equations at the brane are derived.
\end{abstract}

International Workshop on Cosmic Structure and Evolution - Cosmology2009,

September 23-25, 2009

Bielefeld, Germany

\footnotetext{
* Speaker.
} 


\section{Extended theories of gravity}

General Relativity provides a successful classical description of gravitational interactions. Furthermore, it has passed numerous consistency and experimental tests. Nevertheless, it is not without certain shortcomings. For example the resistance towards quantization, or the lack of unification between gauge interactions and gravity, are quite generally recognized as crucial problems. Consequently, an extended theory of gravity is searched for, obviously with Einstein's General Relativity as the main part of its structure, and string theories (or encompassing M-theory) amongst the most serious candidates.

In currently observed 4 space-time dimensions, General Relativity can be regarded as a natural description of gravitational interactions, as the Einstein equation of motion, i.e.

$$
\mathscr{R}_{\mu \nu}-\frac{1}{2} \mathscr{R} g_{\mu \nu}=\kappa T_{\mu \nu}^{(4)}
$$

where $T_{\mu \nu}^{(4)}$ is the energy-momentum tensor and $\kappa=M_{P}^{-2}, M_{P}$ denoting the Planck scale, can be constructed by requiring that the tensor on its 1.h.s. (here: the Einstein tensor) is a most general linear combination of rank 2 tensors fulfilling the conditions for a physically viable theory of gravity. To be more specific, it is (i) symmetric in its indices, (ii) divergence-free (i.e. covariantly conserved), (iii) dependent on the metric tensor and its first two derivatives only, and (iv) linear in the second derivative of the metric tensor. The Einstein equation (1.1) can be also obtained from the Einstein-Hilbert action

$$
\int \mathrm{d}^{4} x \sqrt{-g}\left(\frac{1}{2 \kappa} \mathscr{R}+\mathscr{L}_{M}\right)
$$

which is linear in the Riemann tensor, and $T_{\mu \nu}^{(4)}=g_{\mu \nu} \mathscr{L}_{M}-2 \frac{\delta \mathscr{L}_{M}}{\delta g^{\mu v}}$.

Formulation of string theories requires extra spatial dimensions. In higher-dimensional spacetimes, however, the Einstein tensor is not the only possibility satisfying conditions (i)-(iv), with condition (iv) relaxed to quasi-linearity ${ }^{1}$ in the second derivative of the metric tensor. Specifically, the gravity action (1.2) can be generalized to include certain combinations of higher powers of the Riemann tensor, yielding the so-called Einstein-Lovelock gravity [1]. For a given order in the Riemann tensor the appropriate contribution to the action is unique up to overall normalization, with the quadratic contribution given by the Gauss-Bonnet term [2].

The low energy effective action obtained within the string theories framework, usually referred to in the form of the $\alpha^{\prime}$ expansion, where $\alpha^{\prime}=M_{s}^{-2}$ and $M_{s}$ denotes the string energy scale, actually predicts higher derivative corrections to gravity interactions [3]. To be more explicit, let me address the effective action in string theories restricted to the metric tensor and the dilaton, as these two fields are common for all string theories. At the lowest order of the $\alpha^{\prime}$ expansion, the Einstein-Hilbert Lagrangian (1.2) of the standard gravity is obtained - coupled to the scalar field. The first correction to gravitational interactions can be cast in the form of the Gauss-Bonnet term via local field redefinitions, but higher order corrections appear also for the dilaton interactions. Consequently, higher order theories of gravity coupled to the dilaton seem to be very interesting from the point of view of extended theories of gravity as motivated by string theories.

\footnotetext{
${ }^{1}$ Quasi-linearity in the second derivative of the metric tensor implies that the expression under consideration is still at most of the first order in the second derivative of the metric, but the coefficient of the second derivative can be a function of the first derivative of the metric.
} 


\section{Higher order dilaton gravity}

In [4] the Einstein-Lovelock theory of higher order gravity was generalized to higher order dilaton gravity by coupling to the dilaton. To be more explicit, conditions (i)-(iv) for a physically viable theory of gravity were appropriately extended to accommodate a scalar field interacting with gravity in a higher-dimensional space-time. Specifically, the conditions for the equations of motion

$$
T_{\mu \nu}=\sum_{N=1}^{N_{\max }} \frac{\alpha_{N}}{2} T_{\mu \nu}^{(N)}=0, \quad W=\sum_{N=1}^{N_{\max }} \frac{\alpha_{N}}{2} W^{(N)}=0
$$

of a higher order dilaton gravity theory, where the $N$-th order contributions $T_{\mu \nu}^{(N)}$ and $W^{(N)}$ to the gravitational and the dilaton equations of motion ${ }^{2}$, respectively, involve $2 N$ derivatives of the fields, were established as follows: (I) $T_{\mu \nu}$ is symmetric in its indices, (II) $\nabla_{v} T_{\mu}^{v}=$ const $\cdot\left(\partial_{\mu} \phi\right) W$, (III) $T_{\mu \nu}$ and $W$ depend on the metric, the dilaton and their first two derivatives only, (IV) $T_{\mu \nu}$ and $W$ are quasi-linear ${ }^{3}$ in the second derivatives of both the metric tensor and of the dilaton field, $(\mathrm{V})$ in the string-like frame, where the pure gravity term is multiplied by $e^{-\phi}$, the first derivative of the dilaton appears in the $\left(\partial_{\mu} \phi\right)\left(\partial^{\mu} \phi\right)$ combination exclusively ${ }^{4}$.

Following conditions (I)-(III) and (V), the higher-dimensional equations of motion (2.1) of the higher order dilaton gravity were derived in [4]. Condition (IV) was employed only implicitly and for the metric tensor exclusively - its fulfillment with respect to the dilaton field is a result of the construction. Furthermore, the constructed equations of motion (2.1) are unique up to overall normalization constants $\alpha_{N}$. Moreover, at each step of the construction the number of constraints was higher than the number of available constants, thus the fact that the resulting equations of motion are not vanishing identically is highly non-trivial.

In order to formulate eqs. (2.1) in a particularly compact and tractable form, it is convenient to define the following notation. First of all, let me introduce the generalized Kronecker delta, namely

$$
\delta_{\rho_{1} \rho_{2} \cdots \rho_{N}}^{\sigma_{1} \sigma_{2} \cdots \sigma_{N}}=\operatorname{det}\left[\begin{array}{cccc}
\delta_{\rho_{1}}^{\sigma_{1}} & \delta_{\rho_{2}}^{\sigma_{1}} & \cdots & \delta_{\rho_{N}}^{\sigma_{1}} \\
\cdot & \cdot & \cdots & \cdot \\
\cdot & \cdot & \cdots & \cdot \\
\delta_{\rho_{1}}^{\sigma_{N}} & \delta_{\rho_{2}}^{\sigma_{N}} & \cdots & \delta_{\rho_{N}}^{\sigma_{N}}
\end{array}\right] \text {, }
$$

whose most important property is the full antisymmetry in both covariant and contravariant indices. Basing on (2.2), two generalizations of the standard trace operator can be defined as follows:

$$
\mathscr{T}(M)=\delta_{\rho_{1} \rho_{2} \cdots \rho_{N}}^{\sigma_{1} \sigma_{2} \cdots \sigma_{N}} M^{\rho_{1} \rho_{2} \cdots \rho_{N}} \sigma_{1} \sigma_{2} \cdots \sigma_{N}, \quad \overline{\mathscr{T}}_{\mu}^{v}(M)=\delta_{\mu \rho_{1} \rho_{2} \cdots \rho_{N}}^{v \sigma_{1} \sigma_{2} \cdots \sigma_{N}} M^{\rho_{1} \rho_{2} \cdots \rho_{N}} \sigma_{1} \sigma_{2} \cdots \sigma_{N},
$$

which map tensors of rank $(N, N)$ into numbers or rank $(1,1)$ tensors, respectively. Employing the generalized traces (2.3), raising 'sums' of tensor to a 'power' can be formulated, for example

$$
\begin{aligned}
& \mathscr{T}\left(\left[\frac{1}{2} \mathscr{R}_{* *}^{* *} \oplus 2(\nabla \nabla)_{*}^{*} \phi\right]^{2}\right)=\frac{1}{4} \mathscr{T}\left(\mathscr{R}_{* *}^{* *} \mathscr{R}_{* *}^{* *}\right)+2 \mathscr{T}\left(\mathscr{R}_{* *}^{* *}(\nabla \nabla)_{*}^{*} \phi\right)+4 \mathscr{T}\left((\nabla \nabla)_{*}^{*} \phi(\nabla \nabla)_{*}^{*} \phi\right) \\
& \quad=\frac{1}{4} \delta_{\rho_{1} \rho_{2} \rho_{3} \rho_{4}}^{\sigma_{1} \sigma_{2} \sigma_{3} \sigma_{4}} \mathscr{R}_{\sigma_{1} \sigma_{2}}^{\rho_{1} \rho_{2}} \mathscr{R}_{\sigma_{3} \sigma_{4}}^{\rho_{3} \rho_{4}}+2 \delta_{\rho_{1}}^{\sigma_{1} \sigma_{2} \rho_{3} \sigma_{3}} \mathscr{R}_{\sigma_{1} \sigma_{2}}^{\rho_{1} \rho_{2}}(\nabla \nabla)_{\sigma_{3}}^{\rho_{3}} \phi+4 \delta_{\rho_{1} \rho_{2}}^{\sigma_{1} \sigma_{2}}(\nabla \nabla)_{\sigma_{1}}^{\rho_{1}} \phi(\nabla \nabla)_{\sigma_{2}}^{\rho_{2}} \phi,
\end{aligned}
$$

\footnotetext{
${ }^{2}$ The order $N$ of corrections is restricted by the dimensionality $d$ of space-time. Terms from the $N$-th order contributions $T_{\mu v}^{(N)}$ and $W^{(N)}$ can appear in the equations of motion (2.1) only if $2 N \leq d$.

${ }^{3}$ Quasi-linearity in the second derivatives guarantees that the initial (or boundary) conditions can be formulated in the standard way. This property is also crucial for the existence of brane solutions in the thin wall limit.

${ }^{4}$ Condition (V) restricts quite strongly the form of the constructed equations of motion (2.1), but is a necessary condition of the $O(d, d)$ symmetry expected from string theories (see the discussion of the $O(d, d)$ symmetry in [4]).
} 
where the asterisks ${ }^{5}$ indicate ranks of tensors, $\mathscr{R}_{\mu \nu}^{\rho \sigma} \equiv \mathscr{R}_{\mu v}{ }^{\rho \sigma}$ and $(\nabla \nabla)_{\sigma}^{\rho} \phi \equiv \nabla^{\rho} \partial_{\sigma} \phi$. Thus in the notation of (2.3), the constructed equations of motion (2.1) of higher order dilaton gravity read

$$
\begin{aligned}
& -\sum_{N=1}^{N_{\max }} \frac{\alpha_{N}}{2} \overline{\mathscr{T}}_{\mu \nu}\left(\left[\frac{1}{2} \mathscr{R}_{* *}^{* *} \oplus 2(\nabla \nabla)_{*}^{*} \phi \oplus(-1)(\partial \phi)^{2}\right]^{N}\right)=0, \\
& -\sum_{N=1}^{N_{\max }} \frac{\alpha_{N}}{2} \mathscr{T}\left(\left[\frac{1}{2} \mathscr{R}_{* *}^{* *} \oplus 2(\nabla \nabla)_{*}^{*} \phi \oplus(-1)(\partial \phi)^{2}\right]^{N}\right)=0 .
\end{aligned}
$$

A Lagrangian density corresponding to the constructed equations of motion was also found in [4]:

$$
\mathscr{L}=e^{-\phi}\left\{\sum_{N=1}^{N_{\max }} \frac{\alpha_{N}}{2} \mathscr{T}\left(\left[\frac{1}{2} \mathscr{R}_{* *}^{* *} \oplus 2(\nabla \nabla)_{*}^{*} \phi \oplus(-1)(\partial \phi)^{2}\right]^{N}\right)\right\} .
$$

\section{Higher order dilaton gravity and a co-dimension 1 brane}

The higher order dilaton gravity constructed in [4] is a higher-dimensional theory, and as such it obviously cannot be a straightforward description of our world, as we simply do not observe any extra dimension(s), yet. In order to study the potentially observable predictions of any higherdimensional theory, its effective 4-dimensional description has to be found. The most popular approach in this respect is based on brane scenarios, where the Standard Model is localized on a brane embedded in a higher-dimensional space-time (the 'bulk'), whereas gravity propagates in the full higher-dimensional space-time, as related to the space-time geometry itself. In such a setup a natural question immediately appears: what gravity will be induced at the brane? This problem was addressed in detail in [5], where the effective equations of motion at the brane were derived in the covariant approach ${ }^{6}$, with the bulk action given by the higher order dilaton gravity theory.

The importance of considering the effective gravitational equations at the brane in the higher order dilaton gravity theory should be underlined. This dilaton gravity model is closely related to string theories - its interactions with up to four derivatives are exactly the same as those present in the $\alpha^{\prime}$ expansion in string theories, when restricted to the gravity and the dilaton field (see e.g. [7]). Although such correspondence has not been proven for interactions with six or more derivatives, the theory of higher order dilaton gravity constructed in [4] can be expected to constitute a part of the effective string dilaton gravity action also at the level of more than four derivatives, as is indicated e.g. by the $O(d, d)$ symmetry discussed in [4]. Consequently, the covariant derivation of the effective brane equations in this setup can allow for studying e.g. the potentially observable effects of string theories on specific cosmological models.

At the level of the Lagrangian density, a co-dimension 1 brane corresponds to an inclusion of general brane interactions $\mathscr{L}_{B}$, localized on the brane via Dirac delta type distribution $\delta_{B}$. Hence, allowing also for a bulk scalar potential $V(\phi)$, the Lagrangian density (2.5) becomes

$$
\mathscr{L}=e^{-\phi}\left\{\sum_{N=1}^{N_{\max }} \frac{\alpha_{N}}{2} \mathscr{T}\left(\left[\frac{1}{2} \mathscr{R}_{* *}^{* *} \oplus 2(\nabla \nabla)_{*}^{*} \phi \oplus(-1)(\partial \phi)^{2}\right]^{N}\right)-V(\phi)+\mathscr{L}_{B} \delta_{B}\right\},
$$

\footnotetext{
5 'Powers' and 'sums' $(\oplus)$ of tensors, as well as asterisks, are employed under the generalized traces $\mathscr{T}$ and $\mathscr{T}_{\mu \nu}$ exclusively. For details of notation see [4, 5].

${ }^{6}$ The covariant approach to the derivation of effective brane equations was employed in [6] for the standard gravity, also with the Gauss-Bonnet term or the dilaton included (there, however, the approach was not fully covariant).
} 
whereas the equations of motion (2.4) of the higher order dilaton gravity are modified as follows:

$$
\begin{aligned}
& -\sum_{N=1}^{N_{\max }} \frac{\alpha_{N}}{2} \overline{\mathscr{T}}_{\mu \nu}\left(\left[\frac{1}{2} \mathscr{R}_{* *}^{* *} \oplus 2(\nabla \nabla)_{*}^{*} \phi \oplus(-1)(\partial \phi)^{2}\right]^{N}\right)+g_{\mu \nu} V(\phi)-\tau_{\mu \nu} \delta_{B}=0, \\
& -\sum_{N=1}^{N_{\max }} \frac{\alpha_{N}}{2} \mathscr{T}\left(\left[\frac{1}{2} \mathscr{R}_{* *}^{* *} \oplus 2(\nabla \nabla)_{*}^{*} \phi \oplus(-1)(\partial \phi)^{2}\right]^{N}\right)+V(\phi)-V^{\prime}(\phi)-\tau_{\phi} \delta_{B}=0 .
\end{aligned}
$$

The brane localized terms (sources)

$$
\tau_{\mu v}=h_{\mu v} \mathscr{L}_{B}-2 \frac{\delta \mathscr{L}_{B}}{\delta h^{\mu v}}, \quad \tau_{\phi}=\mathscr{L}_{B}-\frac{\delta \mathscr{L}_{B}}{\delta \phi}
$$

originate from the brane interactions $\mathscr{L}_{B}$, while the metric tensor $h_{\mu \nu}$ induced on the brane is given by a projection of the bulk metric tensor $g_{\mu \nu}$ on the brane hypersurface: $h_{\mu \nu}=g_{\mu \nu}-n_{\mu} n_{v}$, where $n^{\mu}$ is a vector field orthonormal to the brane at its position.

\section{Effective equations of motion at the brane}

Effective brane equations should follow from the bulk equations of motion (3.2) and relate the dynamics of the brane fields (i.e. the induced metric tensor $h_{\mu v}$ and the dilaton field $\phi$, both evaluated on the brane) to the brane localized sources $\tau_{\mu \nu}$ and $\tau_{\phi}$. Optimally, the brane equations should be independent of the bulk solution. It is precisely the latter which implies that the effective $(d-1)$-dimensional equations at the brane cannot be obtained by simply restricting the full $d$ dimensional equations (3.2), as such an approach would leave the result dependent on the bulk quantities. Therefore the derivation of the effective equations of motion at the brane required establishing a quite sophisticated procedure, as it was shown (and approached accordingly) in [5].

In order to derive the effective brane equations of motion, the key bulk quantities appearing in the equations of motion (3.2), i.e. $\mathscr{R}_{\mu \nu}^{\rho \sigma},(\nabla \nabla)_{\rho}^{\sigma} \phi$ and $(\partial \phi)^{2}$, should be replaced with their brane equivalents, namely $R_{\mu \nu}^{\rho \sigma},(D D)_{\rho}^{\sigma} \phi$ and $(D \phi)^{2}$. It should be underlined, that the Riemann tensor $\mathscr{R}_{\mu \nu}^{\rho \sigma}$ and the covariant derivative $\nabla_{\mu}$ are associated with the bulk metric tensor $g_{\mu \nu}$, whereas the Riemann tensor $R_{\mu \nu}^{\rho \sigma}$ and the covariant derivative $D_{\mu}$ - with the brane metric tensor $h_{\mu \nu}$. Due to the full antisymmetrization, most of the appropriate decompositions are considerably simplified under the generalized traces (2.3), yielding

$$
\begin{aligned}
\mathscr{R}_{* *}^{* *} & \rightarrow R_{* *}^{* *}-2 K_{*}^{*} K_{*}^{*}-4(n n)_{*}^{*}\left\{£_{n} K_{*}^{*}-(K K)_{*}^{*}\right\}-8(n D)_{*}^{*} K_{*}^{*}, \\
(\nabla \nabla)_{*}^{*} \phi & \rightarrow\left[(D D)_{*}^{*} \phi+K_{*}^{*} £_{n} \phi\right]+(n n)_{*}^{*}\left\{£_{n}^{2} \phi-a^{e} \nabla_{e} \phi\right\}+2\left[(n D)_{*}^{*} £_{n} \phi-(n K D)_{*}^{*} \phi\right], \\
(\partial \phi)^{2} & =(D \phi)^{2}+\left(£_{n} \phi\right)^{2},
\end{aligned}
$$

where $K_{\mu \nu}=\frac{1}{2} £_{n} h_{\mu \nu}$ is the extrinsic curvature of the brane, $£_{n}$ is the Lie derivative along $n^{\mu}$, and $a^{\lambda} \equiv n^{\rho} \nabla_{\rho} n^{\lambda}$. Moreover, $(n n)_{*}^{*} \equiv n_{*} n^{*},(\nabla \nabla)_{*}^{*} \equiv \nabla_{*} \nabla^{*},(D D)_{*}^{*} \equiv D_{*} D^{*},(K K)_{*}^{*} \equiv K_{\lambda}^{*} K_{*}^{\lambda}$, $(n D)_{*}^{*} \equiv \frac{1}{2}\left(n_{*} D^{*}+n^{*} D_{*}\right)$ and $(n K D)_{*}^{*} \equiv \frac{1}{2}\left(n_{*} K_{\lambda}^{*} D^{\lambda}+n^{*} K_{*}^{\lambda} D_{\lambda}\right)$.

The decompositions (4.1) have to be entered into the bulk equations of motion (3.2). However, not all quantities present on the right hand sides of eqs. (4.1) should appear in the effective equations of motion at the brane. Certainly, brane equations should involve such brane associated quantities 
as $R_{\mu \nu}^{\rho \sigma},(D D)_{\rho}^{\sigma} \phi$ or $(D \phi)^{2}$. On the other hand, neither $K_{\mu \nu}$ nor $£_{n} \phi$ should appear there - nor their Lie derivatives, as all these quantities are derivatives of the brane fields $\left(h_{\mu v}\right.$ and $\left.\phi\right)$ in the direction perpendicular to the brane, thus bulk associated. Moreover, $K_{\mu v}$ and $£_{n} \phi$ can be discontinuous when 'crossing' the brane, hence $£_{n} K_{\mu \nu}$ and $£_{n}^{2} \phi$ can be singular on the brane, though they can have finite contributions as well. Nevertheless, all this information has to be properly taken into account for the derivation of the effective equations of motion at the brane.

\section{Effective equations of motion at the brane in the first order dilaton gravity}

As was demonstrated in detail in [5], the issue of the effective equations of motion at the brane can be addressed in a general way - for any order $N_{\max }$ of corrections appearing in the bulk equations of motion (3.2). Therefore, let me present the outcome for the first order dilaton gravity, i.e. $N_{\max }=1$. Qualitatively, the results and the conclusions are analogous to those for the higher (arbitrary) order dilaton gravity, but the complexity of formulae increases formidably with increasing $N_{\max }$. I will also assume $d=5$, i.e. a 4-dimensional brane.

If no additional assumptions are made about the bulk or the brane, there is just a single brane equation which can be derived, namely

$$
D^{\sigma} \tau_{\rho \sigma}+\left(h_{\rho \sigma} \tau_{\phi}-\tau_{\rho \sigma}\right) D^{\sigma} \phi=0
$$

Moreover, usually it is not even a dynamical equation, unless the brane interactions $\mathscr{L}_{B}$, sourcing (3.3) the brane terms $\tau_{\mu \nu}$ and $\tau_{\phi}$, involve e.g. brane localized kinetic terms for the induced metric tensor $h_{\mu v}$ and/or for the dilaton field $\phi$. Otherwise, eq. (5.1) is just a consistency condition on the brane sources $\tau_{\mu \nu}$ and $\tau_{\phi}$, or a 'generalized' covariant conservation of the energy-momentum tensor $\tau_{\mu \nu}$. Alternatively, eq. (5.1) can be regarded as a relation between the brane derivative of the dilaton field and the brane sources - allowing to determine the dilaton derivative $D_{\mu} \phi$ in terms of $\tau_{\mu \nu}$ and $\tau_{\phi}$. It should be also mentioned that eq. (5.1) has the same simple form also in higher order dilaton gravity - independently of $N_{\max }$.

In brane scenarios it is quite usual by now to 'automatically' assume a $\mathbb{Z}_{2}$ symmetry for the bulk - with its fixed point at the brane position. However, as it was shown in [5], such a $\mathbb{Z}_{2}$ symmetry is actually crucial for the existence of the effective brane equations. With its assumption the first 'true' effective brane equation of motion can be derived, reading

$$
R+2(D D) \phi-(D \phi)^{2}-2 \alpha_{1}^{-1} V(\phi)=\frac{1}{4} \alpha_{1}^{-2}\left[-(\tau \tau)+2 \tau \tau_{\phi}-3 \tau_{\phi}^{2}\right],
$$

where $(\tau \tau) \equiv \tau_{\rho}^{\sigma} \tau_{\sigma}^{\rho}$. Although a single scalar equation certainly cannot be considered a brane analog of the Einstein equation (1.1) for the standard gravity, it can be quite useful e.g. for highly symmetric space-times. Furthermore, combining the effective brane equation (5.2) with the consistency condition (5.1) allows to determine the brane curvature scalar $R$ in terms of the brane sources $\tau_{\mu v}$ and $\tau_{\phi}$ and the value of the potential $V(\phi)$ on the brane.

With the bulk $\mathbb{Z}_{2}$ symmetry assumed, an effective Einstein-like equation at the brane can be also derived, reading

$$
\begin{aligned}
R_{\mu v}-\frac{1}{2} h_{\mu v} R= & -\frac{2}{3}\left(D_{\mu} D_{\nu} \phi-h_{\mu v}(D D) \phi\right)-\frac{1}{4} h_{\mu v}\left((D \phi)^{2}+2 \alpha_{1}^{-1} V(\phi)\right)-E_{\mu v} \\
& +\frac{1}{4} \alpha_{1}^{-2}\left[-(\tau \tau)_{\mu v}+\frac{1}{3} \tau \tau_{\mu v}+h_{\mu v}\left(\frac{1}{2}(\tau \tau)-\frac{1}{12} \tau^{2}-\frac{1}{2} \tau \tau_{\phi}+\frac{3}{4} \tau_{\phi}^{2}\right)\right],
\end{aligned}
$$


where $(\tau \tau)_{\mu \nu} \equiv \tau_{\mu}^{\sigma} \tau_{\sigma \nu}$. Obviously, there are new terms appearing in this equation - as compared to the standard Einstein equation (1.1). First of all, eq. (5.3) involves terms depending on the dilaton field $\phi$, which is typical of gravity theories involving scalar fields. Regarding that it should be supplemented by the consistency condition (5.1), brane derivatives of $\phi$ can be in fact removed. Second, eq. (5.3) depends on the brane sources $\tau_{\mu \nu}$ and $\tau_{\phi}$ quadratically, whereas in the standard gravity the energy-momentum tensor $T_{\mu \nu}^{(4)}$ enters the Einstein equation (1.1) linearly only. Nevertheless, such quadratic contributions are also typical of brane-world gravity theories. Last but not least, the bulk Weyl tensor projected on the brane: $E_{\mu \nu}=n^{\alpha} h_{\mu}^{\beta} n^{\gamma} h_{v}^{\delta} \mathscr{C}_{\alpha \beta \gamma \delta}$, contributing to the Einstein-like effective equation at the brane (5.3). Consequently, the brane dynamics is explicitly influenced by the bulk gravity solution - encoded in a single ${ }^{7}$ quantity. Summarizing, although an Einstein-like effective equation at the brane (5.3) can be obtained if the bulk $\mathbb{Z}_{2}$ symmetry is assumed, it is generically bulk-dependent. The scalar effective equation at the brane (5.2) and the consistency condition (5.1) are the only bulk-independent brane equations which can be derived.

Terms linear in the energy-momentum tensor (present in the standard Einstein equation (1.1)) can be reintroduced into the Einstein-like brane equation of motion (5.3) by performing the following decomposition: $\tau_{\mu v}=\widetilde{\tau}_{\mu \nu}+h_{\mu \nu} \tilde{\lambda}$, where $\widetilde{\tau}_{\mu \nu}$ is the energy-momentum tensor associated with the fields we are interested in (e.g. the Standard Model fields assumed to be localized on the brane), and $\widetilde{\lambda}$ - a 'cosmological constant'. Consequently, the Einstein-like brane equation (5.3) becomes

$$
\begin{aligned}
R_{\mu v}-\frac{1}{2} h_{\mu v} R & =8 \pi \widetilde{G} \widetilde{\tau}_{\mu \nu}-\frac{2}{3}\left((D D)_{\mu \nu} \phi-h_{\mu v}(D D) \phi\right)-\frac{1}{4} h_{\mu v}(D \phi)^{2}-h_{\mu v} \widetilde{\Lambda} \\
& -E_{\mu \nu}+\frac{1}{4} \alpha_{1}^{-2}\left[-(\widetilde{\tau} \widetilde{\tau})_{\mu v}+\frac{1}{3} \widetilde{\tau} \widetilde{\tau}_{\mu \nu}+h_{\mu \nu}\left(\frac{1}{2}(\widetilde{\tau} \widetilde{\tau})-\frac{1}{12} \widetilde{\tau}^{2}+\left(\frac{2}{3} \widetilde{\lambda}-\frac{1}{2} \tau_{\phi}\right) \widetilde{\tau}\right)\right]
\end{aligned}
$$

where

$$
\widetilde{G} \equiv \frac{-(d-3) \widetilde{\lambda}}{32(d-2) \pi \alpha_{1}^{2}}, \quad \widetilde{\Lambda}=\left.\frac{1}{2} \alpha_{1}^{-1} V(\phi)\right|_{\phi=0}-\frac{1}{4} \alpha_{1}^{-2}\left(\widetilde{\lambda}^{2}-\left.2 \tilde{\lambda} \tau_{\phi}\right|_{\phi=0}+\left.\frac{3}{4} \tau_{\phi}^{2}\right|_{\phi=0}\right)
$$

can be interpreted as the effective brane Newton's and cosmological constants, respectively.

\section{Modified Friedmann equations at the brane}

All the results presented above were obtained in the covariant approach, without any additional assumptions on the brane or the bulk solutions - apart from the bulk $\mathbb{Z}_{2}$ symmetry, which is essential for the existence of effective brane equations. However, studying the details of the effective 4-dimensional phenomenology requires at least a specific ansatz on the brane metric tensor. According to the standard cosmological picture, confirmed by numerous observations, our expanding world can be to a good approximation described as spatially homogeneous and isotropic, which corresponds to a maximally spatially symmetric space-time. Consequently, the Friedmann-LemaîtreRobertson-Walker (FLRW) ansatz can be assumed for the brane metric tensor $h_{\mu \nu}$, to be followed by appropriate assumptions (basically corresponding to homogeneity and isotropy) for other relevant quantities: $E_{\mu}^{v}=\operatorname{diag}\left(E_{t}^{t},-\frac{1}{3} E_{t}^{t},-\frac{1}{3} E_{t}^{t},-\frac{1}{3} E_{t}^{t}\right), D_{\mu} \phi=(\dot{\phi}, 0,0,0), \tau_{\mu}^{v}=\operatorname{diag}\left(\tau_{t}, \tau_{i}, \tau_{i}, \tau_{i}\right)$.

\footnotetext{
${ }^{7}$ Note that there is no direct dependence on the bulk scalar solution.
} 
The effective gravitational equation at the brane in the first order dilaton gravity (5.3) can be treated as a modified Einstein equation. Hence, evaluating it for the FLRW ansatz leads to modified Friedmann equations, namely

$$
\begin{aligned}
& \frac{\dot{a}^{2}}{a^{2}}+\frac{k}{a^{2}}= \frac{2}{3} \frac{\dot{a}}{a} \dot{\phi}-\frac{1}{12} \dot{\phi}^{2}+\frac{1}{6} \alpha_{1}^{-1} V(\phi)+\frac{1}{3} E_{t}^{t} \\
&+\frac{1}{16} \alpha_{1}^{-2}\left[\frac{1}{3} \tau_{t}^{2}-\tau_{i}^{2}-\frac{2}{3} \tau_{t} \tau_{i}+\frac{2}{3} \tau_{t} \tau_{\phi}+2 \tau_{i} \tau_{\phi}-\tau_{\phi}^{2}\right], \\
& \frac{\ddot{a}}{a}+\frac{\dot{a}^{2}}{a^{2}}+\frac{k}{a^{2}}=\frac{\dot{a}}{a} \dot{\phi}-\frac{1}{6} \dot{\phi}^{2}+\frac{1}{3} \ddot{\phi}+\frac{1}{3} \alpha_{1}^{-1} V(\phi) \\
&+\frac{1}{24} \alpha_{1}^{-2}\left[-\tau_{t}^{2}-3 \tau_{i}^{2}+2 \tau_{t} \tau_{\phi}+6 \tau_{i} \tau_{\phi}-3 \tau_{\phi}^{2}\right],
\end{aligned}
$$

where $a(t)$ denotes the cosmological scale factor, and $k$ is the space curvature constant. Furthermore, the Friedmann equations (6.1) should be supplemented by the consistency condition (5.1) evaluated for the FLRW ansatz, reading

$$
\dot{\tau}_{t}+3 \frac{\dot{a}}{a}\left(\tau_{t}-\tau_{i}\right)+\dot{\phi}\left(\tau_{\phi}-\tau_{t}\right)=0 .
$$

The modified Friedmann equations (6.1) (with the consistency condition (6.2) taken into account) include the standard terms involving the scale factor $a(t)$, but also a functional dependence on the brane sources $\tau_{\mu \nu}$ and $\tau_{\phi}$ (instead of the components of the energy-momentum tensor entering linearly the standard Friedmann equations) and their time derivatives, as well as the direct bulk influence encoded in $E_{\mu \nu}$. Regarding that their solution yields the evolution of the scale factor governing the expansion of the universe, the effective cosmology at the brane will be modified with respect to the standard picture. Therefore solving the effective Friedmann equations for the scale factor $a(t)$ will allow comparisons between the predictions of the first order dilaton gravity in the brane scenario and the experimental limits given by cosmological observations, thus constituting a step towards understanding the phenomenological implications of the string theory motivated dilaton gravity.

\section{References}

[1] D. Lovelock, J. Math. Phys. 12 (1971) 498.

[2] C. Lanczos, Annals Math. 39 (1938) 842.

[3] B. Zwiebach, Phys. Lett. B 156 (1985) 315; D.G. Boulware and S. Deser, Phys. Rev. Lett. 55 (1985) 2656; Phys. Lett. B 175 (1986) 409; R. R. Metsaev and A. A. Tseytlin, Phys. Lett. B 191 (1987) 354; D. J. Gross and J. H. Sloan, Nucl. Phys. B 291 (1987) 41.

[4] D. Konikowska and M. Olechowski, Phys. Rev. D 76 (2007) 124020 [arXiv:0704.1234[hep-th]].

[5] D. Konikowska and M. Olechowski, [arXiv:0908.1052[hep-th]].

[6] M. Sasaki, T. Shiromizu and K. i. Maeda, Phys. Rev. D 62 (2000) 024008 [arXiv:hep-th/9912233]; T. Shiromizu, K. i. Maeda and M. Sasaki, Phys. Rev. D 62 (2000) 024012 [arXiv:gr-qc/9910076]; K. i. Maeda and T. Torii, Phys. Rev. D 69 (2004) 024002 [arXiv:hep-th/0309152]; K. i. Maeda and D. Wands, Phys. Rev. D 62 (2000) 124009 [arXiv:hep-th/0008188].

[7] K. A. Meissner, Phys. Lett. B 392 (1997) 298 [arXiv:hep-th/9610131]. 